\title{
A Performance Comparison of Automatic Detection Schemes in Wide-Area Aerial Imagery
}

\author{
Xin Gao, Sundaresh Ram, and Jeffrey J. Rodríguez \\ Department of Electrical and Computer Engineering, The University of Arizona, Tucson, AZ, USA \\ Email: \{xgao1985, ram, jjrodrig\}@email.arizona.edu
}

\begin{abstract}
Accurate and efficient detection of vehicles in wide-area aerial imagery is a fundamental task in understanding the automobile traffic patterns in an urban environment so as to help regulate the traffic flow. Vehicles with varying shapes and sizes, background clutter, occlusion, low-resolution and noise in the acquired images make the automatic detection of vehicles a challenging task. We present the performance analysis of six object detection algorithms for moving vehicle detection in low-resolution aerial image sequences. We compare the automatic detection results with manual detection, and evaluate the performance of the six object detection algorithms via several metrics.
\end{abstract}

Index Terms-Object detection, wide-area aerial imagery.

\section{INTRODUCTION}

The development of robust and efficient object detection algorithms has received widespread interest in the field of image analysis. In particular, detecting moving objects in wide-area aerial imagery has a wide range of applications, from visual surveillance to security related tasks such as rescue and evacuation [1], [12]. Compared to the on-road vehicle detection studies [11], detecting vehicles from widearea aerial imagery offers much broader visual coverage. Many applications such as traffic surveillance require robust and accurate object detection as the primary processing step; however, due to the low contrast, appearance model diversity, varying motion, and object occlusion, the task of object detection has often been a difficult problem, especially when handling low-resolution datasets. In this paper, we investigate six algorithms for object detection. We have applied each of the detection algorithms to two low-resolution aerial video datasets. The detection performance is evaluated by matching the automated detection results to the ground-truth vehicles. Finally, the overall performance on both datasets is quantified by the metrics of precision, recall, F-score, and percentage of wrong classifications.

\section{DATASETS AND ALGORITHMS}

We used two aerial video datasets obtained from a lowresolution video camera $(720 \times 480$ pixels $)-$ the first one covers a Tucson local traffic scene (vehicle area ranging from 40 to 150 pixels), and the other is a view of a freeway near Phoenix (vehicle area ranging from 20 to 175 pixels). Each video was manually cropped to extract just the traffic lanes so that we could focus on the vehicle detection problem. (Road extraction may also be performed automatically using existing road detection algorithms or using GIS data if available.) A manual segmentation serves as the ground truth, which includes 4012 and 4060 rectangular-shaped vehicles for 100 consecutive frames for the Tucson dataset and the Phoenix dataset, respectively.

Six object detection algorithms motivated by previously published works were evaluated using the two datasets. The key details of each method are summarized below.

1) The spectrum residual (SR) model: The SR object detection algorithm was proposed by Hou and Zhang [2]. In this method, the salient objects within the image are detected by analyzing the log-spectrum of the input image and extracting the spectral residual of the image, which is the difference between the log-spectrum and the averaged spectrum in the spectral domain. Next, the algorithm computes a saliency map corresponding to the spectral residual in the spatial domain by taking the inverse Fourier transform. Finally, objects are detected by thresholding the saliency map, which is smoothed by a Gaussian lowpass filter with $\sigma=8$. To adapt this method to our low-resolution aerial images, we use a Gaussian filter with $\sigma=0.5$.

2) Segmentation with variational minimax optimization (VMO): Saha and Ray [3] developed a locally adaptive image thresholding technique for segmentation, using variational energy optimization. This method automatically computes the weights on the data fidelity and the regularization terms in the variational formulation by combining them in a convex non-linear fashion and obtains an optimum threshold surface via the minimax principle. To apply the VMO segmentation technique to the vehicle detection problem, we modify the threshold surface by multiplying it with a threshold scaling factor of 0.7 to achieve a reasonable balance between false positives and missed vehicles.

3) Fuzzy logic (FL) vehicle detection: Jain and Rani [4] derived an algorithmic approach using fuzzy logic to detect vehicles. Their FL algorithm has three stages: (i) initial processing to construct sixteen probabilistic logic matrices 
that represent intensity differences between two neighboring pixels; (ii) a defuzzification step to compute a normalized maximum weight for each pixel; (iii) a final fuzzy logic thresholding on the normalized edge map, followed by a morphological fill operation for region segmentation. For the Tucson dataset, we choose a membership function $(\mathrm{a}, \mathrm{b}, \mathrm{c})=$ $(0.3,0.8,0)$ to initialize the probability matrix in stage (i) and 0.1 for thresholding in stage (iii); for the Phoenix dataset, we use a membership function $(a, b, c)=(0.3,0.6,0)$ in stage (i) and 0.2 for thresholding in stage (iii).

4) Feature density estimation (FDE): Gleason et al. [5] proposed a vehicle detection method by estimating density of the vehicle features. Their method comprises four stages. The first stage of this algorithm inspects every image location at various scales and eliminates the background areas by detecting features using the Sobel edge detector. In the second stage, areas containing high density of features (i.e., feature values above a certain threshold) are detected. Since the second stage returns a large number of overlapping areas around actual vehicles, in the third stage they are grouped using a clustering scheme. In the final stage, color-based properties are used to refine the results and precisely detect the vehicle locations. To adapt this method to our datasets, we select a grayscale threshold of 50 for binary conversion in stage two of this method, and we do not perform the third and fourth stages.

5) Morphological filtering (MF): Zheng et al. [6] developed an automatic vehicle detection algorithm using mathematical morphology. This method comprises the following steps: (i) application of grayscale opening followed by grayscale top-hat transformation and grayscale closing followed by grayscale bottom-hat transformation on the original image to concurrently detect positive- and negativecontrast vehicles, (ii) Otsu's thresholding on the resultant image to identify the probable vehicles in the image. To adapt this method for our datasets, we use a $3 \times 3$ square structuring element for all the morphological operations [10] in this algorithm.

6) Subpixel location with partial area effects (SL-PAE): Trujillo-Pino et al. [7] proposed a method to detect the edges in images with subpixel accuracy. This method initially hypothesized a non-continuous model for image acquisition. The image acquisition model is derived from partial area effects, such as subpixel position, orientation, curvature, and change in intensity values on both sides of the edge. To adapt this method to our datasets, we use a grayscale value of 28 to specify the minimum difference of intensity at both sides of a pixel to be considered as an edge.

Post-processing: The six object detection algorithms as described above generate a high rate of false detections on each frame. In order to reduce the false detections of all the algorithms, we use the following post-processing method: an object sieving process is performed to retain all the binary objects in every frame which have object area $A \in\left[t_{\text {low }}, t_{\text {high }}\right]$. We select these thresholds as $[5,160]$ and $[5,180]$ for the Tucson dataset and the Phoenix dataset, respectively. This object sieving process significantly reduces false positives in both datasets for the SR, VMO and FDE approaches. For the remaining three detection algorithms, FL, MF, and SL-PAE, the sieving process is followed by a morphological closing operation with a $3 \times 3$ uniform structuring element.

\section{ERROR CLASSIFICATION}

To evaluate the object detection algorithms [2]-[7], we assign the detections using 8-connected component labeling, and evaluate the overlap between detection and true object in a way similar to the region matching procedure by Nascimento [8], where we characterize detections as follows:

True positive (TP): correct detection. If multiple detections intersect the same ground truth object, then only one TP is counted (the one having largest overlap). If a single detection intersects multiple ground truth objects, then only one TP is counted (the one having largest overlap);

False negative $(\mathrm{FN})$ : detection failure indicated by a ground truth object that does not intersect any detected object;

False positive (FP): incorrect detection indicated by a detection that does not intersect any ground truth object.

\section{EXPERIMENTAL RESULTS}

In this section, we exhibit the performance analysis of the six detection algorithms. Table I displays the total number of classified detections for each algorithm in chronological order, and the last column shows the publication year of the original references.

TABLE I

Classification of Detections for EaCh Method in 100 Frames

\begin{tabular}{|c|c|c|c|c|c|}
\hline \multirow{2}{*}{ Algorithm } & Dataset & TP & FN & FP & \multirow{2}{*}{ Year } \\
\hline \multirow{2}{*}{ SR } & Tucson & 3321 & 537 & 900 & \multirow{2}{*}{2007} \\
\cline { 2 - 5 } & Phoenix & 2045 & 1768 & 1591 & \\
\hline \multirow{2}{*}{ VMO } & Tucson & 3735 & 84 & 6412 & \multirow{2}{*}{2009} \\
\cline { 2 - 5 } & Phoenix & 3439 & 422 & 4076 & \\
\hline \multirow{2}{*}{ FL } & Tucson & 3404 & 231 & 2254 & \multirow{2}{*}{2010} \\
\cline { 2 - 5 } & Phoenix & 2967 & 978 & 6900 & \\
\hline \multirow{2}{*}{ FDE } & Tucson & 3375 & 613 & 2694 & \multirow{2}{*}{2011} \\
\cline { 2 - 5 } & Phoenix & 2804 & 977 & 2280 & \multirow{2}{*}{2013} \\
\hline \multirow{2}{*}{ MF } & Tucson & 3453 & 136 & 1668 & \multirow{2}{*}{2013} \\
\cline { 2 - 5 } & Phoenix & 2262 & 1585 & 1439 & \\
\hline \multirow{2}{*}{ SL-PAE } & Tucson & 3205 & 560 & 1621 & \multicolumn{2}{|c|}{} \\
\cline { 2 - 5 } & Phoenix & 2613 & 968 & 1603 & \\
\hline
\end{tabular}

Comparison of TPs and FNs (misses): VMO exhibits the highest total of TPs, MF ranks the second best in the Tucson dataset, while VMO and FL perform the highest and the second highest in TPs in the Phoenix dataset, respectively. The sum of TPs in the other five algorithms are close (with SL-PAE displaying the lowest sum of TPs) in the Tucson dataset, while the high-to-low rank is FL, FDE, SL-PAE, MF and SR for TPs in the Phoenix dataset. For the Tucson dataset, all the algorithms have average FN rates lower than $10 \mathrm{FNs}$ 
per frame. For the Phoenix dataset, VMO, FL, FDE and SLPAE have average FN rates lower than $10 \mathrm{FNs}$ per frame.

Comparison of FPs: For the Tucson dataset, SR has the lowest total of FPs, while SL-PAE and MF rank the second, the third lowest, and VMO performs the poorest. For the Phoenix dataset, MF has the fewest FPs, SR and SL-PAE rank the second and the third best, while FL has the most FPs.

Fig. 1 depicts a visual comparison of original frames with visual vehicles, detections from each algorithm (color boundary on regions) and the ground truth (rectangular regions in white color).

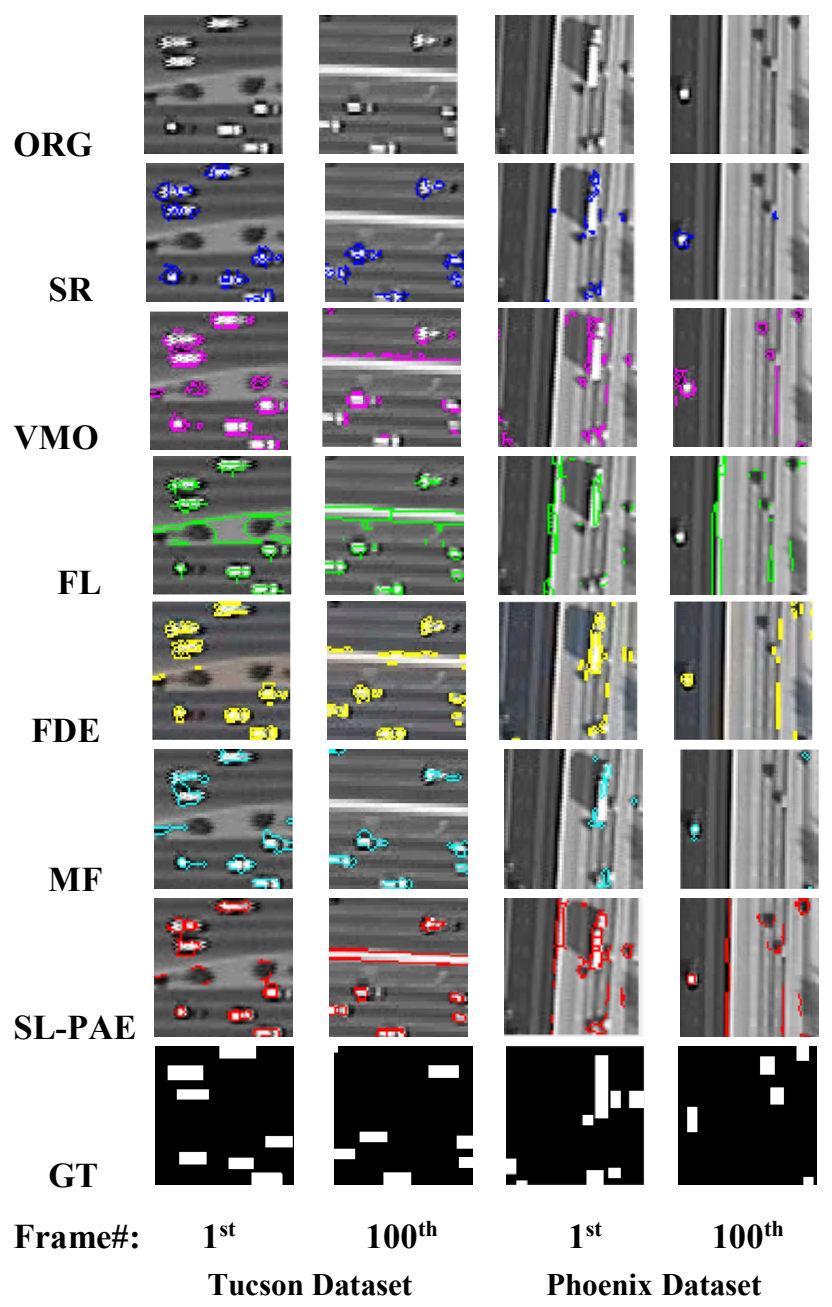

Fig. 1. Visual comparison of vehicle detections for the six algorithms. The first row displays original frames (ORG); the last row exhibits ground truth (GT). Subimage size: $64 \times 64$ pixels.

We employ a set of evaluation metrics [9] that comprehensively measure the detection performance:

$$
\begin{aligned}
\text { Precision } & =\frac{\mathrm{TP}}{\mathrm{TP}+\mathrm{FP}} \\
\text { Recall } & =\frac{\mathrm{TP}}{\mathrm{TP}+\mathrm{FN}}
\end{aligned}
$$

$$
\text { F-Score }=\frac{2 \times \text { Precision } \times \text { Recall }}{\text { Precision }+ \text { Recall }}
$$

where F-score is the harmonic mean of precision and recall.

The percentage of wrong classification (PWC) [9] evaluates the ratio of the sum of FNs and FPs, to the total number of all four types of detections:

$$
\mathrm{PWC}=\frac{\mathrm{FN}+\mathrm{FP}}{\mathrm{TP}+\mathrm{FN}+\mathrm{FP}+\mathrm{TN}}
$$

Since we have no negative samples on detection, $\mathrm{TN}=0$.

Quantitative comparisons for the six object detection algorithms are charted in Fig. 2. We also present statistics for each evaluation metric (t-test with $95 \%$ confidence intervals among the data for 100 frames for each dataset) in Table II. For the Tucson dataset, SR displays the best precision, Fscore and PWC, while VMO performs the poorest under the three metrics but the highest recall. For the Phoenix dataset, SL-PAE performs the best on precision, F-score and PWC, SR displays the lowest recall, the second poorest F-score and PWC, MF has the second best precision but the second lowest recall, VMO displays the highest recall and second lowest precision, and FL performs the second best in recall and the poorest on precision, F-score and PWC.
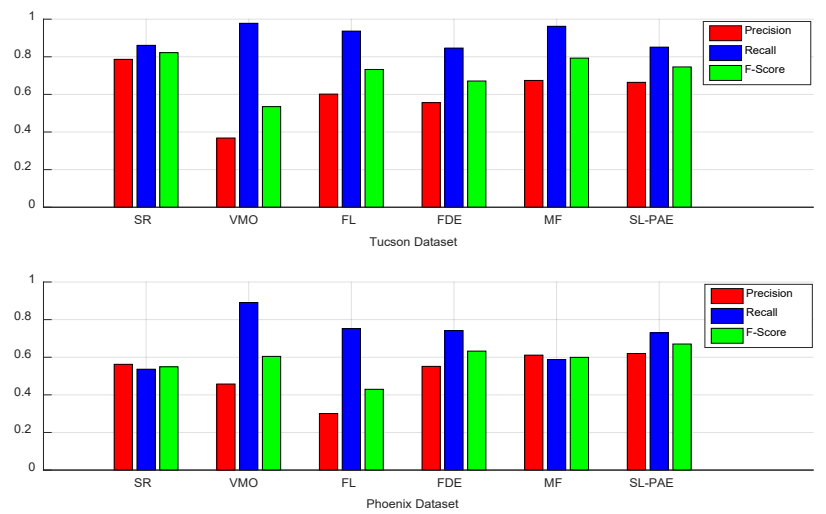

Fig. 2. Quantitative measures of precision, recall, and F-score for the six detection algorithms.

In sum, VMO, FL and MF have recalls of above 0.9 in the Tucson dataset, but only VMO has a recall close to 0.9 in the Phoenix dataset. Each algorithm has the same rank of precision and F-score in the Tucson dataset, while SR, VMO, FL and MF rank differently in the Phoenix dataset; however, for each algorithm, recall rate is higher in the Tucson dataset than in the Phoenix dataset, but the ranks of each metric have no consistent pattern between the two datasets. The rank of PWC (from high to low) for each algorithm in each dataset coincides with the rank of F-score (from low to high), and this is further supported by the tight confidence intervals for these metrics. 


\section{CONCLUSION}

We have presented a comprehensive analysis of six object detection algorithms using low-resolution image sequences. For the specific task of vehicle detection in widearea aerial imagery, the performance comparison indicates that VMO represents the best algorithm on recall for both datasets. FL and MF achieve recall rates higher than 0.9. SR, FDE and SL-PAE have a recall rate close to 0.85 in the Tucson dataset. However, each algorithm achieves lower recall rate in the Phoenix dataset, where VMO displays the highest recall rate, which is close to 0.9. Regarding the evaluation metrics of precision, F-score, and PWC, the ranks of each algorithm are identical in the Tucson dataset (with SR displaying the best scores on each metric), while exhibiting variable differences (with SL-PAE displaying the best scores on each metric) in the Phoenix dataset.

\section{ACKNOWLEDGMENT}

The authors are grateful to Prof. Mark Hickman, School of Civil Engineering, The University of Queensland, Australia, for providing the aerial video datasets.

\section{REFERENCES}

[1] R. Porter, A. M. Fraser, and D. Hush, "Wide-area motion imagery," IEEE Signal Process. Mag., vol. 27, no. 5, pp. 56-65, Sep. 2010.

[2] X.-D. Hou and L.-Q. Zhang, "Saliency detection: a spectral residual approach," in Proc. IEEE Conf. Comput. Vis. Pattern Recognit. (CVPR), Jun. 18-23, 2007, Minneapolis, MN, pp. 1-8.

[3] B. N. Saha and N. Ray, "Image thresholding by variational minimax optimization," Pattern Recognit., vol. 42, no. 5, pp. 843-856, May 2009.

[4] I. Jain and B. Rani, "Vehicle detection using image processing and fuzzy logic," Int. J. Comput. Sci. Commun., vol. 1, no. 2, pp. 255-257, Jul.-Dec. 2010.
[5] J. Gleason, A. V. Nefian, X. Bouyssounousse, T. Fong, and G. Bebis, "Vehicle detection from aerial imagery," in 2011 IEEE Int. Conf. Robot. Automat. (ICRA), May 9-13, 2011, Shanghai, China, pp. 2065-2070.

[6] Z.-Z. Zheng, G.-Q. Zhou, Y. Wang, Y.-L. Liu, X.-W. Li, X.-T. Wang, and L. Jiang, "A novel vehicle detection method with high resolution highway aerial image," IEEE J. Sel. Top. Appl. Earth Observ. Remote Sens., vol. 6, no. 6, pp. 2338-2343, Dec. 2013.

[7] A. Trujillo-Pino, K. Krissian, M. Alemán-Flores, and D. Santana-Cedrés, "Accurate subpixel edge location based on partial area effect," Imag. Vis. Comput., vol. 31, no. 1, pp. 7290, Jan. 2013.

[8] J. C. Nascimento and J. S. Marques, "Performance evaluation of object detection algorithms for video surveillance," IEEE Trans. Multimedia, vol. 8, no. 4, pp. 761-774, Aug. 2006.

[9] S. H. Shaikh, K. Saeed, and N. Chaki. Moving Object Detection using Background Subtraction, Springer, 2014, pp. 30-31.

[10] S. Li, G.-Q. Zhou, Z.-Z. Zheng, Y.-L. Liu, X.-W. Li, Y. Zhang, and T. Yue, "The relation between accuracy and size of structure element for vehicle detection with high resolution highway aerial images," in 2013 Int. Geosci. and Remote Sens. Symp. (IGARSS), Jul. 21-26, 2013, Melbourne, Australia, pp. 2645-2648.

[11] S. Sivaraman and M. M. Trivedi, "Looking at vehicles on the road: a survey of vision-based vehicle detection, tracking, and behavior analysis," IEEE Trans. Intell. Transp. Syst., vol. 14, no. 4, pp. 1773-1795, Dec. 2013.

[12] R. C. Philip, S. Ram, X. Gao, and J. J. Rodríguez, "A comparison of tracking algorithm performance for objects in wide area imagery," in 2014 IEEE Southwest Symp. Image Anal. Interp. (SSIAI), Apr. 6-8, 2014, San Diego, CA, pp. 109112.

TABLE II

Performance Evaluation of Object Detection Algorithms

(MEAN AND 95\% CONFIDENCE INTERVAL; LEFT: TUCSON DATASET, RIGHT: PHOENIX DATASET)

\begin{tabular}{|c||c|c|c|c||c|c|c|c|}
\hline Algorithm & Precision & Recall & F-Score & PWC \% & Precision & Recall & F-Score & PWC \% \\
\cline { 2 - 9 } SR & $\begin{array}{c}0.7903 \\
\pm 0.0118\end{array}$ & \begin{tabular}{c}
0.8608 \\
\multirow{2}{*}{$\mathrm{SRO}$}
\end{tabular} & $\begin{array}{c}0.8226 \\
\pm 0.0086\end{array}$ & $\begin{array}{c}29.88 \\
\pm 1.23\end{array}$ & $\begin{array}{c}0.5809 \\
\pm 0.0192\end{array}$ & $\begin{array}{c}0.5363 \\
\pm 0.0132\end{array}$ & $\begin{array}{c}0.5497 \\
\pm 0.0091\end{array}$ & $\begin{array}{c}61.96 \\
\pm 0.86\end{array}$ \\
\hline \multirow{2}{*}{$\mathrm{VMO}$} & 0.3730 & 0.9781 & 0.5387 & 63.01 & 0.4605 & 0.8909 & 0.6061 & 56.41 \\
& \pm 0.0084 & \pm 0.0047 & \pm 0.0090 & \pm 0.84 & \pm 0.0080 & \pm 0.0074 & \pm 0.0073 & \pm 0.75 \\
\hline \multirow{2}{*}{$\mathrm{FL}$} & 0.6069 & 0.9364 & 0.7345 & 41.71 & 0.3024 & 0.7516 & 0.4306 & 72.50 \\
& \pm 0.0131 & \pm 0.0047 & \pm 0.0100 & \pm 1.27 & \pm 0.0061 & \pm 0.0099 & \pm 0.0071 & \pm 0.58 \\
\hline \multirow{2}{*}{$\mathrm{FDE}$} & 0.5602 & 0.8465 & 0.6728 & 49.15 & 0.5585 & 0.7416 & 0.6347 & 53.27 \\
& \pm 0.0104 & \pm 0.0075 & \pm 0.0085 & \pm 0.97 & \pm 0.0146 & \pm 0.0102 & \pm 0.0110 & \pm 1.20 \\
\hline \multirow{2}{*}{$\mathrm{MF}$} & 0.6831 & 0.9624 & 0.7959 & 33.53 & 0.6223 & 0.5874 & 0.6002 & 56.74 \\
& \pm 0.0161 & \pm 0.0069 & \pm 0.0115 & \pm 1.54 & \pm 0.0194 & \pm 0.0151 & \pm 0.0143 & \pm 1.49 \\
\hline \multirow{2}{*}{$\mathrm{SL}-\mathrm{PAE}$} & 0.6671 & 0.8513 & 0.7471 & 40.25 & 0.6258 & 0.7296 & 0.6711 & 49.26 \\
& \pm 0.0090 & \pm 0.0079 & \pm 0.0071 & \pm 0.90 & \pm 0.0153 & \pm 0.0096 & \pm 0.0105 & \pm 1.19 \\
\hline
\end{tabular}

